December 1, 1992 through February 28, 1993

Project Title:

Principal Investigator:

Project Manager:
Mass spectral study of organic Bulfur in the polymeric Matrix of Coal

DE-FC22-92PC92521

Luke Hanley, Assistant Professor

Department of Chemistry, m/c 111

University of Illinois at Chicago

Chicago, IL 60607-7061

Ken Ho, ICCI

\title{
AB8TRACT
}

This report reviews the sixth quarter progress of a two year project to examine the chemical environment of organic sulfur in the polymeric matrix of Illinois coal by laser desorption ion trap mass spectrometry. This project is attempting to develop new laser desorption-ionization schemes for coal which preserve the polymeric matrix.

During this quarter, we extended experiments utilizing ultraviolet matrix assisted laser desorption ionization to the examination of solvent extracts of coal. Regardless of the matrix compound, concentration, or laser wavelength chosen, only carbon cluster ions were observed in the mass spectra. During the next quarter, we will attempt to apply laser desorption of neutral compounds followed chemical ionization to form intact molecular ions from the polymeric component of coal.

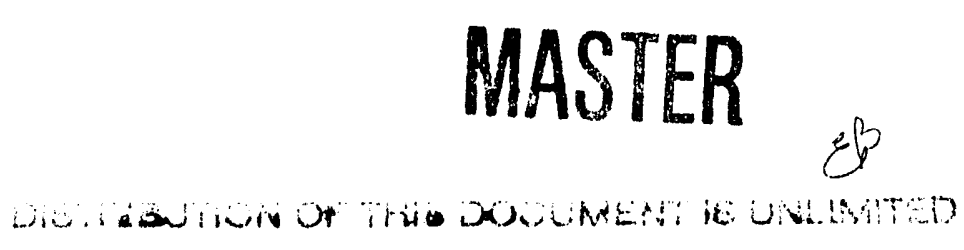

"U.S. DOE Patent Clearance is NOT required prior to the publication of this document." 


\section{EXECUTIVE BUMEARY}

Organically bound sulfur in coal, part of which resides in the polymeric matrix, is perhaps the most significant impediment to the economical utilization of Illinois coal. It follows that the elucidation of the chemical environment of organic sulfur in the polymeric matrix would assist the development of chemical, microbial, and enzymatic treatments for sulfur removal from Illinois coal. This project is attempting to apply the new technique of laser desorption ion trap mass spectrometry to the analysis of organic sulfur in this polymeric matrix.

During the last quarter, we developed the capability to perform ultraviolet matrix assisted laser desorption ionization (UVMALDI) of solvent extracts of coal. In this method, polymeric species are dissolved in a solution of an ultraviolet light absorbing matrix compound, such as 2,5dihydroxybenzoic acid. This sample is then dried onto a stainless steel probe tip, placed into vacuum, and desorbed using an ultraviolet laser pulse. UVMALDI has been recently implemented in many laboratories around the world for the desorption and ionization of a wide variety of involatile and labile polymers and biopolymers. At the start of this quarter, we demonstrated by collecting spectra of a calibration compound, bovine insulin, that the mass range of UVMALDI experiments in our apparatus is $\sim 6,000 \mathrm{~m} / \mathrm{z}$.

During the remainder of this quarter, we have attempted to apply UVMALDI to the desorption and ionization of solvent extracts of coal. Solvent extracts of Illinois No. 6 coal were obtained by soxhlet extraction in pyridine since previous reports have indicated that coal polymers can be extracted in this manner. A wide variety of matrix compounds were utilized, including 2,5-dihydroxybenzoic acid, nicotinic acid, nitrophenyl octyl ether, sinapinic acid, and nitrobenzyl alcohol. Cation attachment compounds such as potassium chloride and silver trifluoroacetate were added to enhance ion formation. The samples wexe prepared in a variety of concentration ranges and desorption was attempted with both $266 \mathrm{~nm}$ and $532 \mathrm{~nm}$ radiation.

Despite these extensive efforts, no intact molecular ions representative of the polymeric matrix of coal were observed in UVMALDI mass spectra. Carbon clusters were the only high mass positive or negative ions detected from coal solvent extracts in any of these experiments.

We have begun implementing another method for the mass analysis of coal polymers which involves laser desorption of neutrals followed by cation attachment of $\mathrm{Li}^{+}$ions. All of the necessary parts have been assembled for these desorption chemical ionization experiments, which will be discussed in the next quarterly report. 


\section{OBJECTIVES}

These experiments are attempting to examine the chemical environment of organic sulfur in the polymeric matrix of Illinois coal by laser desorption of high molecular weight fragments into an ion trap mass spectrometer. If we can successfully laser desorb intact molecular ions from the coal polymeric matrix, then we will test the validity of the maceral separation method for the analysis of coal by comparing the mass spectra of different macerals to determine whether the separation process also chemically alters the coal samples.

The milestones scheduled to be completed during this, the second year of the project, are as follows:

Task 1: Application of solvent extraction to coal macerals to produce chemically simplified samples for mass spectra analysis via laser desorption.

Task 2: Comparison of laser desorption mass spectra for whole Illinois No. 6 coal with several of its separated macerals.

Task 3: Sulfur determination of separated coal macerals via collision induced dissociation.

Task 4: Further sulfur determination of separated coal macerals via high resolution laser desorption mass spectrometry. Data analysis and preparation of final conclusions for publication. below.

We are still working on Task 1 , as will be discussed

\section{INTRODUCTION AND BACKGROUND}

organically bound sulfur in coal, part of which resides in the polymeric matrix, is perhaps the most significant impediment to the economical utilization of Illinois coal. It follows that the elucidation of the chemical environment of organic sulfur in the polymeric matrix would assist the development of chemical, microbial, and enzymatic treatments for sulfur removal from Illinois coal. This project seeks to apply the new technique of laser desorption ion trap mass spectrometry to the analysis of organic sulfur in this polymeric matrix.

It has been found that the analysis of organic sulfur in separated macerals of coal is much simpler than the direct analysis of whole coal. Macerals are separated by the density gradient centrifugation process, which is assumed to not alter the overall polymeric and chemical structure from that in whole coal. While it has been shown that model 
compounds are unaffected by the density gradient centrifugation process, it is entirely possible that these compounds are not accurate representations of the complex and unexpected chemical processes which might occur in whole coal. Given the present emphasis on maceral separation, these experiments strive to provide an independent method of verifying the absence of chemical modification during this process.

\section{EXPERIMENTAI PROCEDUREB}

Solvent extracts of solid coal are deposited onto a steel probe and placed in the vacuum chamber, where they are exposed to ultraviolet laser pulses. Ions which desorb from the surface are injected into an ion trap mass spectrometer for mass analysis. Further instrumental details can be found in previous quarterly reports. In the next several paragraphs, we will discuss the details of sample preparation for ultraviolet matrix assisted laser desorption (UVMALDI) of both a high mass calibration compound and for solvent extracts of coal.

To test the upper mass limit of ions generated by UVMALDI, a sample of a calibration compound, bovine insulin, was prepared by dissolving $2.6 \mathrm{mg}$ of the protein in a $0.1 \%$ aqueous trifluoroacetic acid solution. This protein/acid solution was then added to another solution of 2,5dihydroxybenzoic acid (DHB)/d-fructose solution to make a 5000:5000:1 molar ratio mixture of DHB/d-fructose/bovine insulin. The resulting solution was applied to a stainless steel probe tip and allowed to dry in air.

Solvent extracts were prepared as discussed in the literature: The pyridine soluble portion of Illinois No. 6 coal was isolated via soxhlet extraction procedures at $-115^{\circ} \mathrm{C}$ under a positive pressure of argon gas. The excess pyridine was removed from the extract by gentle heating under reduced pressure. To limit the amount of oxidation by air, the resulting dark brown solid extract was stored under argon until needed. The coal extract solution used for UVMALDI samples was prepared by dissolving a $4.6 \mathrm{mg}$ portion of the extract in $3 \mathrm{ml}$ of pyridine.

The matrix compounds used for preparation of UVMALDI samples of coal solvent extracts were chosen based on compatibility with pyridine and the UV laser wavelength available in our lab, $266 \mathrm{~nm}$. Based on these considerations, nicotinic acid, nitrophenyl octyl ether (NPOE), and sinapinic acid were chosen. The solid matrix materials were dissolved directly in pyridine to make suitable solutions. A $2.5 \times 10^{-4}$ molar sinapinic acid solution was made by dissolving $28 \mathrm{mg}$ of acid in $5 \mathrm{ml}$ pyridine. $A \sim 4 \times 10^{-4}$ molar nicotinic acid solution was prepared in a similar manner. 
Samples for the UVMALDI experiments were made by adding the extract solution to the matrix solutions on stainless steel probe tips and allowing the solvent to evaporate. For each different matrix, a broad range of matrix/analyte solutions were used since the true molecular weight of the extracted material was not known. Additional experiments were performed where potassium chloride and silver trifluoroacetate were added to the sample in attempts to facilitate cation attachment.

The experiments employing NPOE as the matrix used solutions prepared using a slightly different procedure since NPOE is a liquid at room temperature. Portions of the coal extract from $1 \mathrm{mg}$ to $5 \mathrm{mg}$ were added to $1 \mathrm{ml}$ NPOE. The solution was allowed to turn a brown color over a day to ensure the dissolution of the extract material in the matrix. The brown solution was then added to a stainless steel probe tip as usual. Again, metal salts were added in an attempt to produce cation-attached species.

\section{REBULTS AND DI8CO88ION}

During the fifth quarter, we developed the capability to perform ultraviolet matrix assisted laser desorption ionization (UVMALDI) on a calibration compound of approximately 1,000 Da molecular weight. Because this method has been demonstrated to be so effective for the generation of intact molecular ions from a wide range of labile, high molecular weight biomolecules and polymers, we thought it might also be a effective for coal polymers.

We began this quarter by running experiments to verify that we could desorb, ionize, and detect high mass ions via UVMALDI: The resultant mass spectrum of a calibration compound, bovine insulin $(5733 \mathrm{~m} / \mathrm{z})$, is shown below in Figure 1. Approximately 5 nanomoles of bovine insulin were loaded onto the probe tip for this spectrum. An intact molecular ion peak can be seen at $-5700 \mathrm{~m} / \mathrm{z}$ along with a peak arising from the ionized chain $B$ portion of the molecule, indicating our ability to detect ions up to $\sim 6000 \mathrm{~m} / \mathrm{z}$ via UVMALDI.

once the parameters for producing and trapping large molecules using the UVMALDI technique were optimized, the rest of the quarter was spent applying the technique to samples of coal solvent extracts. The positive ion spectra of a $3: 1 \mathrm{v} / \mathrm{v}$ mixture of nicotinic acid solution/coal extract solution is shown in Figure 2. All of the individual ion peaks correspond to carbon clusters and the largest peak at $\sim 720 \mathrm{~m} / \mathrm{z}$ corresponds to the exceptionally stable $\mathrm{C}_{60}{ }^{+}$ion known popularly as buckyball. Even at the moderate power densities used for UVMALDI experiments, $10^{6}-10^{7} \mathrm{w} / \mathrm{cm}^{2}$, carbon clusters such as the ones shown could be seen in the 
spectra. Negative carbon cluster ions were also detected during UVMALDI. Figure 3 shows a low mass range spectra of a $20: 1 \mathrm{v} / \mathrm{v}$ sinapinic acid solution/coal extract solution. Carbon clusters were the only high mass positive or negative ions detected from any of the different matrices used for sample preparation.

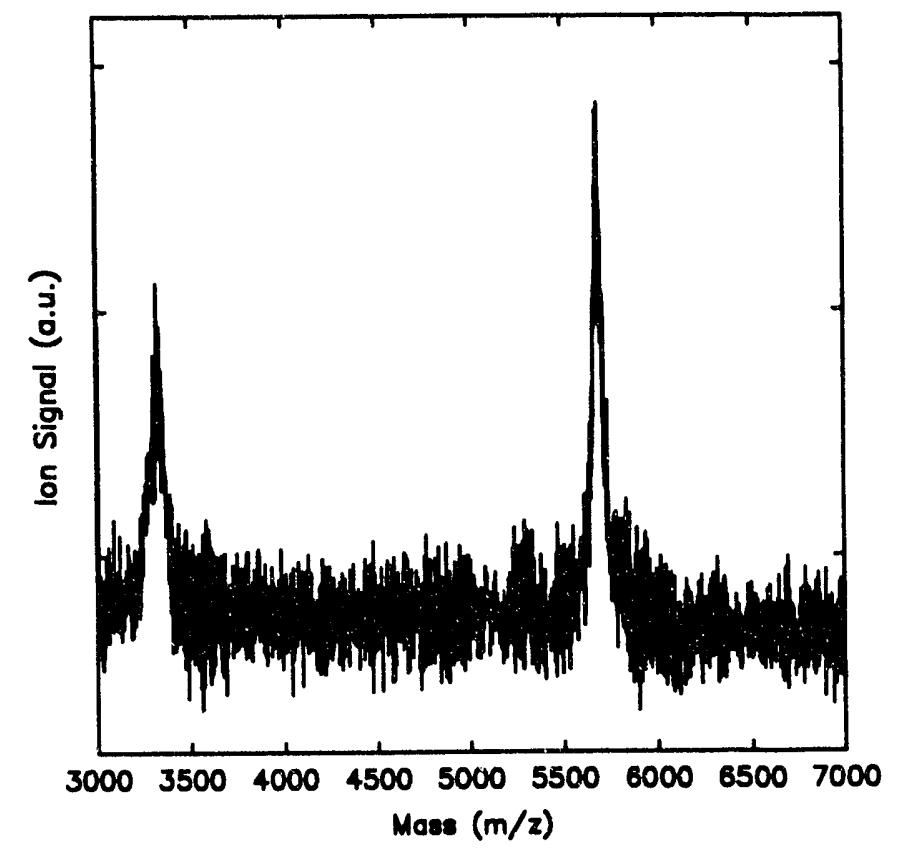

Figure 1: UVMALDI mass spectrum of a calibration compound, bovine insulin.

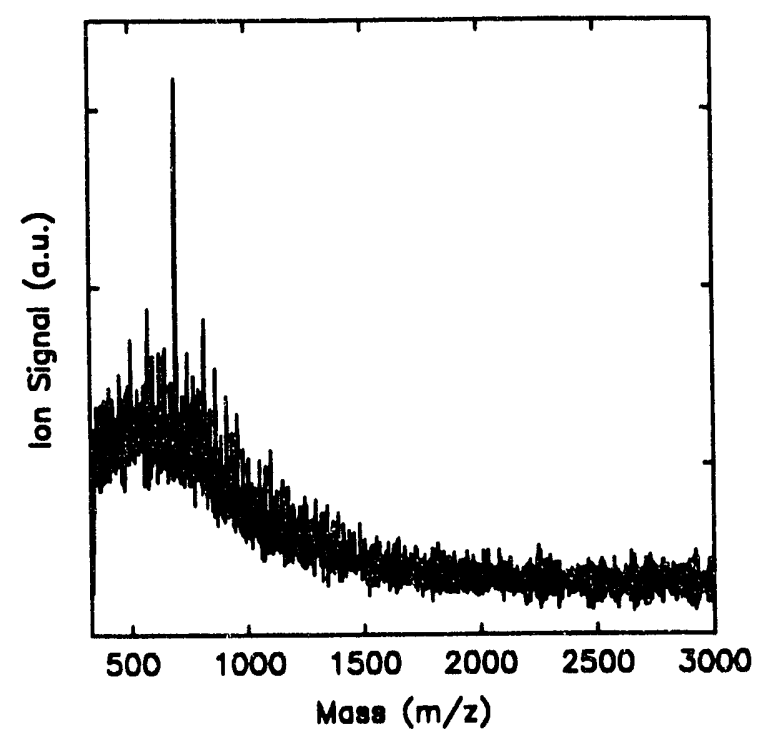

Figure 2: UVMALDI positive ion mass spectrum of pyridine solvent extract from Illinois No. 6 coal. 


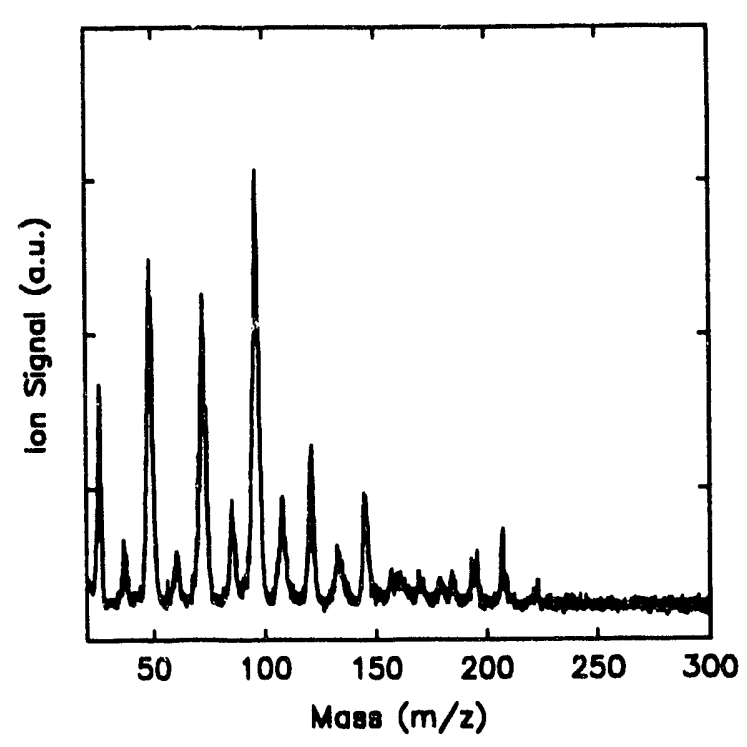

Figure 3: UVMALDI negative ion mass spectrum of pyridine solvent extract from Illinois No. 6 coal.

It is known that the aromatic nature of coal will result in strong optical absorption of the $266 \mathrm{~nm}$ radiation used in UVMALDI. It appears that direct photoabsorption by the coal extracts leads to significant photochemically induced rearrangement resulting in the formation of carbon clusters. To remedy this problem, we have attempted matrix assisted laser desorption ionization with visible wavelengths $(532 \mathrm{~nm})$, utilizing Rhodamine-6G dye and nitrobenzyl alcohol as a matrix. Unfortunately, no ions whatsoever were observed which could be attributed to the coal extract.

We have one final laser desorption/ionization scheme which we can apply to form intact molecular ions from coal. This method involves laser desorption of neutrals followed by cation attachment of $\mathrm{Li}^{+}$ions in a process known as desorption chemical ionization. All of the necessary parts have been assembled for these experiments, which will be discussed in the next quarterly report. 


\section{CONCLUSIONS AND RECOMMENDATIONS}

1) Ultraviolet matrix assisted laser desorption ionization of solvent extracts of coal has not been successful in generating intact molecular ions of the polymeric matrix of coal. Once again, only carbon clusters were formed and these are thought to result from photochemical rearrangement.

2) Matrix assisted laser desorption ionization of solvent extracts of coal using visible radiation did not produce any ions whatsoever from the coal sample.

3) We are now attempting to implement chemical ionization combined with laser desorption to produce intact molecular ions from solvent extracts of coal. The results of these experiments will appear in the next quarterly report. 
PROJECT MANAGEMENT REPORT

December 1, 1992 through February 28, 1993

Project Title:

Mass spectral study of Organic sulfur in the polymeric Matrix of $\operatorname{coal}$

Principal Investigator: Luke Hanley, Assistant Professor Department of Chemistry, m/c 111 University of Illinois at Chicago Chicago, IL 60607-7061

Project Manager: Ken Ho, ICCI

\section{COMMENTS}

The slightly higher than estimated materials \& supplies costs result from the implementation of new experimental configurations last quarter (UVMALDI) and again this quarter (chemical ionization). These costs are offset by lower than expected direct labor expenditures. The higher than estimated other direct costs result from expenses occurring earlier in the project year than initially estimated. The graduate student, John Burroughs, and the undergraduate, Brian Cadre, continue to work on the project. The iess than expected travel costs will be offset by higher costs in this category during the third quarter. A budget change request will be submitted in June to reflect the above variations from initial estimates. However, this budget change request will not alter the total cost of the project. 


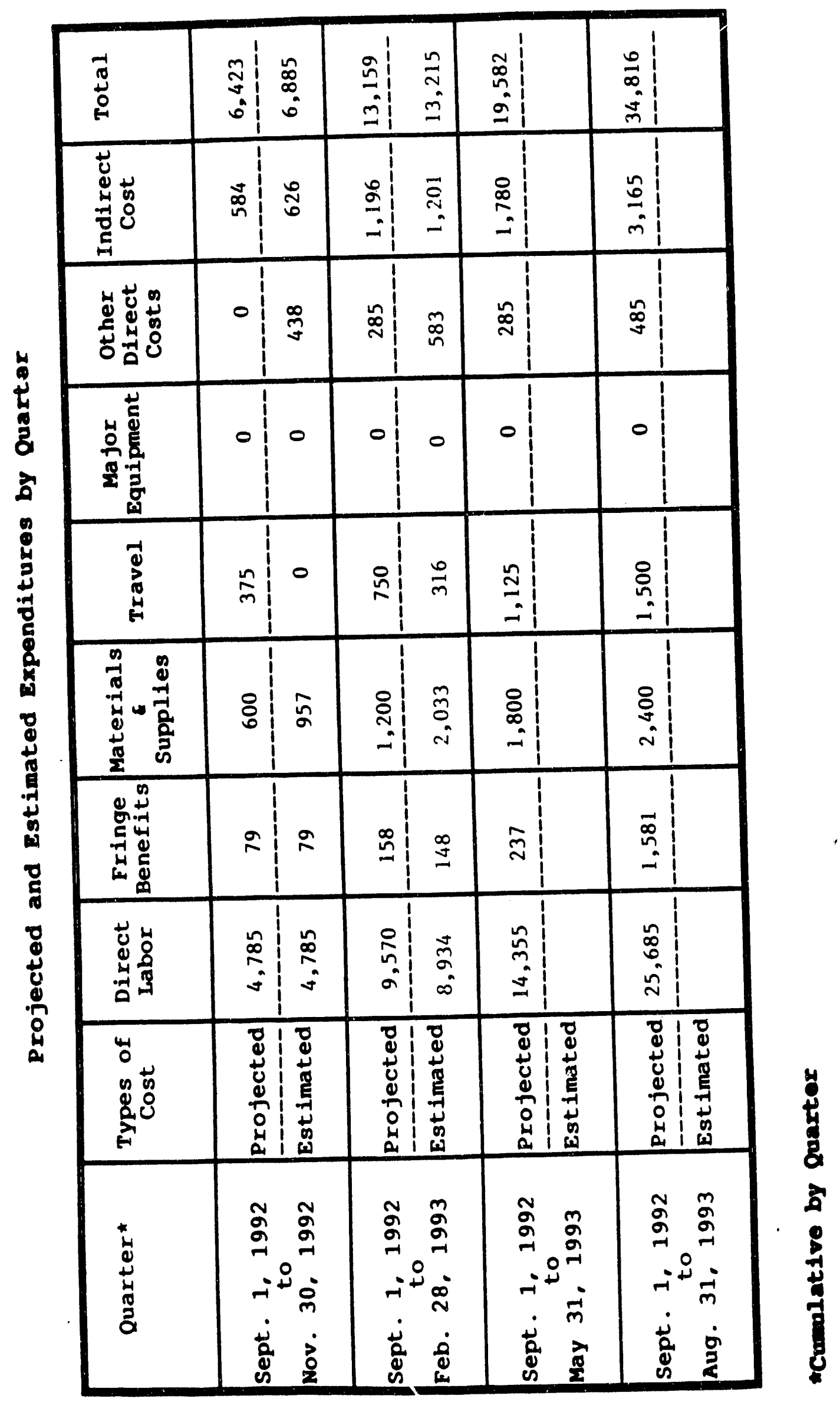


COSTS BY QUARTER

(Mass spectral study of organic sulfur

in the Polymeric Matrix of Coal)

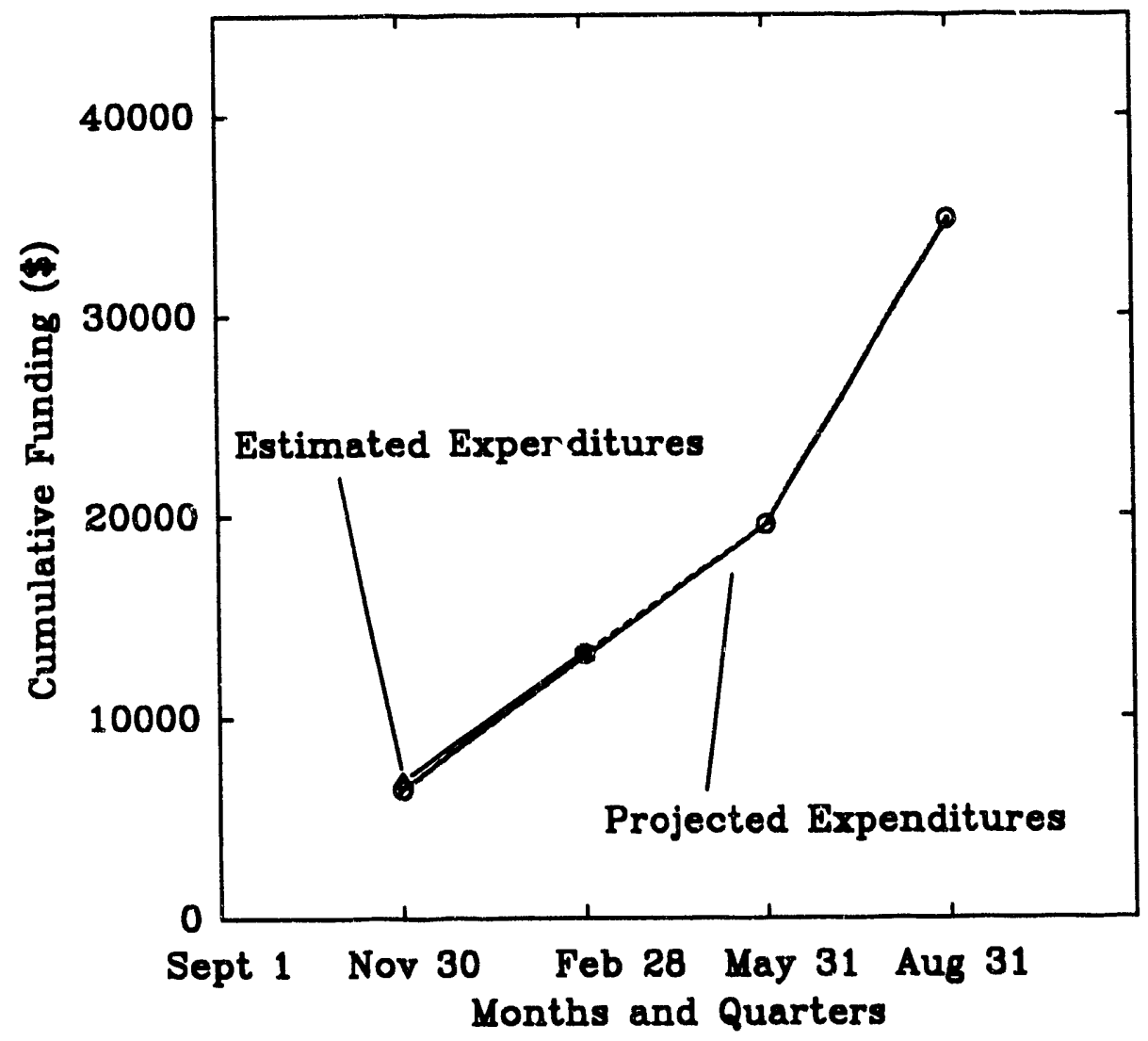

Total CRSC Award $\$ 34,816$ 
8CHEDULE OF PROJECT MILESTONES

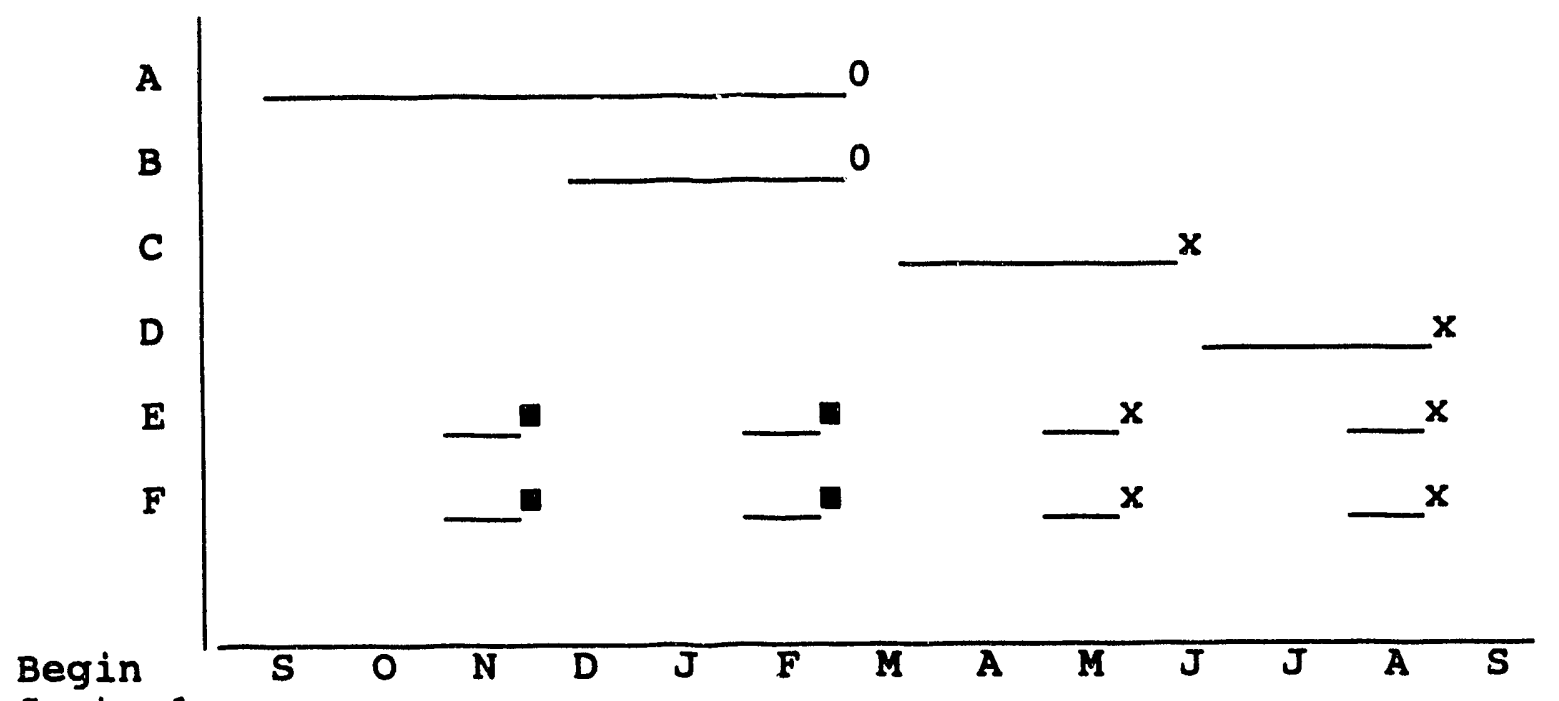

Sept. 1

1992

Milestones:

A. Application of solvent extraction to coal macerals to produce chemically simplified sampies for mass spectra analysis via laser desorption. (Task 1).

B. Comparison of laser desorption mass spectra for whole Illinois No. 6 coal with several of its separated macerals. (Task 2).

C. Sulfur determination of separated coal macerals via collision induced dissociation. (Task 3).

D. Further sulfur determination of separated coal macerals via high resolution laser desorption mass spectrometry. Data analysis and preparation of final conclusions for publication. (Task 4).

E. Technical reports prepared and submitted.

F. Project management reports prepared and submitted.

Comments: We are still working on obtainin high mass spectra from the solvent extracts of coal, as is discussed in the technical report. 
DATE FILMED $6 / 21 / 93$ 
